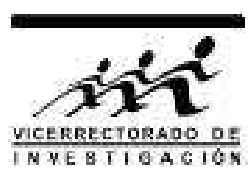

\title{
Determinación del rendimiento hídrico en la cuenca alta del río Grande en Puno
}

\author{
Douglas D. Sarango, Guido A. Rozas, Bernardino Salvador, Teresa O. Velásquez, Jaime Sullca y \\ Julio J. Mansilla
}

Facultad de Ciencias Físicas, Universidad Nacional Mayor de San Marcos, Lima 1, Perú

Recibido 16 noviembre 2012 - Aceptado 15 marzo 2012

\begin{abstract}
A partir del año 2000 se viene realizando intensivamente la explotación de oro por las cooperativas, las empresas mineras y las asociaciones clandestinas que desarrollan minería informal en la cuenca alta del río Grande, distrito de Ananea, Puno. Estas actividades producen residuos y desechos mineros que se sedimentan y generan impactos ambientales negativos. Una forma de remediar estos impactos se produce mediante la construcción de una presa para almacenar y retener estos sedimentos. En este sentido, para poder diseñar la presa y determinar los volúmenes de sedimentos a ser almacenados, es necesario determinar una serie de caudales medios mensuales en el sitio del eje de la presa. En la cuenca alta del río Grande no se cuenta con estaciones hidrométricas o hidrográficas que permitan medir el aporte hídrico de la cuenca, pero la precipitación ocurrida es medida en la estación climatológica de Ananea. El presente trabajo usa un modelo de precipitación - escorrentía para la determinación del recurso hídrico obteniéndose como resultado una serie de caudales medios mensuales. Los resultados muestran un caudal promedio anual de $3.22 \mathrm{~m}^{3} / \mathrm{s}$ y un volumen promedio anual de 100.07 MMC en el sitio de emplazamiento de la presa a implementarse en la parte alta de la cuenca del río Grande.

Palabras claves: Cuenca del río Grande, impactos ambientales, sedimentos, presa, precipitación, caudales medios mensuales.
\end{abstract}

\section{Determination of hidric performance in the high basin of Grande river in Puno}

Since 2000, different cooperatives, mining enterprises and also some forms of informal clandestine exploitation have been performing heavy minery of gold in the high basin of Rio Grande, of the district of Ananea-Puno. The environmental impact comes from the residues or waste released from the mining activity, being the sediments the ones that cause it. Ways to solve this is impacts is through the construction of a dam to storage and hold these residues. In this sense, in order to design the dam and determine the volumes of sediments to be storaged, it was necessary to determine a series of monthly streamflow at the dam site axis. The high basin of Rio Grande doesn't count with hydrometric o hydrographic stations, but precipitation occurred is measuring at Ananea's climatology station. Because of this summary the present investigation uses precipitation - streamflow model to determine streamflows, using as data monthly precipitation and obtaining monthly streamflow. The results shown annual average flow of $3.22 \mathrm{~m}^{3} / \mathrm{s}$ and the annual average volume of 100.07 MMC at the dam site axis proyected on the higher part of the basin of river Grande.

Keywords: Basin of river Grande, environmental impact, sediments, dam, precipitation, monthly streamflows.

La intensa actividad minera relacionada con la explotación del oro ha tenido un gran impacto ambiental negativo en el valle del río Grande, desde la cuenca alta del río Grande en la zona de Ananea en la región de Puno hasta su desembocadura en el lago Titicaca [1,2]. Este gran impacto ambiental es producido por los residuos y desechos que se liberan en la gran actividad minera en el distrito de Ananea y que se sedimentan a lo largo del río Grande hasta el lago Titicaca provocando un impacto negativo en la biodiversidad del valle y el lago, simultáneamente. Una forma de remediar y disminuir el impacto negativo es la construcción de una presa para almacenar y retener estos sedimentos.

Para diseñar la presa y determinar los volúmenes de los sedimentos a ser almacenados es necesario determinar una serie de los caudales mensuales medios en el 
sitio del eje de la presa, además se tiene presente que en la cuenca alta del río Grande, Ananea, Puno, no se cuenta con las estaciones hidrométricas e hidrográficas que permiten medir el aporte hídrico de la cuenca, pero sí se tienen los registros de la precipitación ocurrida a lo largo de los años anteriores en la estación climatológica de Ananea.

Con estas informaciones proponemos una metodología para la determinación de los recursos hídricos en las cuencas que poseen escasa información hidrométrica. El detalle de la metodología lo desarrollamos a continuación.

La hipótesis que se plantea en el presente trabajo es que la generación de los caudales mensuales medios a partir de la información de la precipitación total mensual permite determinar los caudales mensuales en un punto específico de una cuenca que carece de los registros de los caudales mensuales medios, lo que permitirá la gestión y el manejo de los recursos hídricos para la solución de los problemas existentes en la cuenca.

\section{El modelo de Lutz Scholz}

Entre los modelos estocásticos y markovianos, el modelo desarrollado por Lutz Scholz 345 para las cuencas de la sierra peruana, entre los años 1979-1980, en el marco de la cooperación técnica de la República de Alemania con el Perú a través del Plan Meris II, es el más conocido. Este modelo hidrológico mixto combina una estructura determinística para el cálculo de los caudales mensuales y para el año promedio basado en el balance hídrico de los modelos determinísticos y una estructura estocástica para la generación de las series extendidas del caudal mediante los procesos Markovianos del modelo estocástico.

El modelo se desarrolló tomando en consideración parámetros físicos y meteorológicos de las cuencas, que pueden ser obtenidos a través de las mediciones cartográficas y de campo. Los parámetros más importantes del modelo son la determinación de la precipitación efectiva, el déficit de escurrimiento, la retención y agotamiento de las cuencas. Los procedimientos que se han seguido en la implementación del modelo son [1]: (a) el cálculo de los parámetros necesarios para la descripción de los fenómenos de escorrentía promedio, (b) el establecimiento de un conjunto de modelos parciales de los parámetros para el cálculo de los caudales en las cuencas sin información hidrométrica, (c) la calibración del modelo y la generación de los caudales extendidos por un proceso markoviano combinado de precipitación efectiva del mes con el caudal del mes anterior.

\section{Coeficiente de Escurrimiento}

En la cuenca que no dispone de registros hidrométricos que permiten un cálculo del coeficiente de escorrentía de manera directa, este parámetro se puede calcular aplicado el método de L. Turc. Según este método el coeficiente de escorrentía $C$ depende de la precipitación $P$ promedio anual en $\mathrm{mm} / \mathrm{año}$ y del déficit de escurrimiento $D$ en $\mathrm{mm}$ /año, que a su vez depende de una función de la temperatura $L$, siendo la temperatura media anual $T$ en ${ }^{\circ} \mathrm{C}$, es decir,

$$
C=\frac{P-D}{P},
$$

donde

$$
D=\frac{P}{\left(0.9+\frac{P^{2}}{L^{2}}\right)^{0.5}}
$$

y

$$
L=300+25 T+0.05 T^{3} .
$$

\section{La generación de caudales mensuales medios en cuenca $\sin$ registros}

Para la generación de caudales mensuales medios en cuencas sin registros hidrométricos usamos el modelo de Lutz Scholz, para ello consideramos la precipitación total mensual en mm, el área de la cuenca en estudio en $\mathrm{km}^{2}$, la retención de la cuenca $R$ en $\mathrm{mm}$, definida como la lámina de la lluvia retenida por la cuenca y que contribuye al abastecimiento de agua en la época de estiaje. Esta lámina se ha calculado a partir de los acuíferos potenciales como las lagunas y los nevados que de acuerdo a la pendiente de la cuenca retiene una determinada lámina de agua. El coeficiente de agotamiento $a$ se obtiene a partir de la fórmula empírica de Moss, como una función del área de la cuenca $A$ e interviene en el cálculo de los caudales en época de estiaje,

$$
a=-0.00252 \ln (A)+0.030 \text {. }
$$

La generación de los caudales comprende $a$ ) el cálculo de la precipitación efectiva $P_{E}$ con el registro de la precipitación promedio mensual $P$ mediante el método de la FAO,

Tabla 1: Rango de aplicación de la precipitación efectiva por el método de la FAO.

\begin{tabular}{cc}
\hline$P(\mathrm{~mm})$ & $P_{E}(\mathrm{~mm})$ \\
\hline 0 & 0 \\
10 & 0 \\
20 & 2 \\
$<75$ & $P_{E}=0.6 P-10$ \\
$>75$ & $P_{E}=0.8 P-25$ \\
\hline
\end{tabular}

b) el cálculo de la retención hídrica $R_{i}$ de las lagunas, de los pantanos y de la napa freática en la cuenca analizada; c) el cálculo del gasto de la retención $G_{i}$ desde el 
mes de abril hasta el mes de octubre, (I) considerando que la relación entre la descarga del presente mes y el mes anterior está dado por

$$
g_{0}=e^{-a t}
$$

donde $a$ es el coeficiente de agotamiento y $t$ es número de días de un mes que consideramos igual a 30 días; (II) la relación de descarga del presente mes y el mes anterior para la estación seca se obtiene mediante

$$
g_{i}=\left(g_{0}\right)^{i}
$$

donde $i=1, \ldots, 7$ considerando desde el mes de abril hasta octubre, respectivamente; (III) y el cálculo del gasto de la retención de los meses de la estación seca está dado por

$$
G_{i}=\frac{R_{i} g_{i}}{\sum_{i=1}^{6} g_{i}}
$$

d) el cálculo del abastecimiento de la retención $A_{i}$ del mes $i$ que se presenta en la estación lluviosa entre octubre y marzo dado por

$$
A_{i}=a_{i} R_{i}
$$

donde $a_{i}$ es el coeficiente de abastecimiento; e) el cálculo de la generación de caudales mensuales $C M_{i}$ para el año promedio que se obtiene mediante

$$
C M_{i}=P_{E i}+G_{i}-A_{i} ;
$$

f) con los resultados obtenidos en el ítem $e$ ) se efectúa la regresión lineal múltiple entre el caudal del mes $Q_{t}$, el caudal del mes anterior $Q_{t-1}$ y la precipitación efectiva del mes $P_{E t}$, determinándose los coeficientes de regresión, el error estándar y el coeficiente de correlación; g) luego, se calcula de la precipitación efectiva mensual de todo el registro de precipitación mensual; $h$ ) además la generación de los números aleatorios con distribución normal con media cero y variancia igual a $1, \mathrm{y} i$ ) finalmente, con los datos de los ítems $e), f), g$ ) se procede a la generación de los caudales medios mensuales mediante

$$
Q_{t}=b_{1}+b_{2} Q_{t-1} b_{3} P E_{t}+S\left(1-R^{2}\right)^{1 / 2} Z_{t i}
$$

donde $Q_{t}$ y $Q_{t-1}$ son los caudales generados en el año $t$ y $t-1$, respectivamente, $P_{E t}$ es la precipitación efectiva del año $t, S$ es el error standard y $Z$ es el número aleatorio normal $(0,1)$ del año $t$ y $b_{1}, b_{2}, b_{3}$ son los coeficientes de la regresión multiple.

\section{Análisis del problema}

La información utilizada corresponde a la cuenca alta del río Ramis, donde toma el nombre de río Grande, es el afluente más importante de los que desembocan en el Lago Titicaca, se extiende desde los $5828 \mathrm{msnm}$ en el nevado Ananea hasta los 3815 msnm, ver Fig.1.

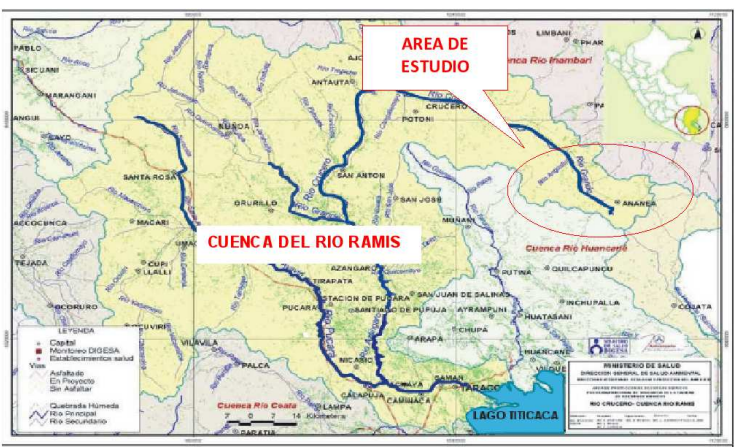

Figura 1: Cuenca del río Ramis, Fuente: Ministerio de Salud.

El área de las lagunas 6 de la cuenca alta del río Grande en la zona de Ananea es aproximadamente de $27.13 \mathrm{~km}^{2}$. Dentro de la cuenca alta del río Grande en

\begin{tabular}{|c|c|c|c|c|c|c|c|c|c|}
\hline \multirow[b]{2}{*}{ Cuenca } & \multirow[b]{2}{*}{ Nombre } & \multirow[b]{2}{*}{$\begin{array}{c}\text { Perímetro } \\
(\mathrm{km})\end{array}$} & \multirow[b]{2}{*}{$\begin{array}{c}\text { Área } \\
\left(\mathrm{km}^{2}\right)\end{array}$} & \multicolumn{2}{|c|}{ Rectángulo equivalente } & \multirow[b]{2}{*}{$\begin{array}{c}L \\
(\mathrm{~km})\end{array}$} & \multirow[b]{2}{*}{$\begin{array}{l}L_{C G} \\
(\mathrm{~km})\end{array}$} & \multirow{2}{*}{$\begin{array}{c}\text { Factor de } \\
\text { forma } \\
\left(F=A / L^{2}\right)\end{array}$} & \multirow{2}{*}{$\begin{array}{c}\text { Índice de } \\
\text { compacidad } \\
K=0.28 P / A\end{array}$} \\
\hline & & & & $\begin{array}{c}L^{\prime} \\
(\mathrm{km})\end{array}$ & $\begin{array}{c}l^{\prime} \\
(\mathrm{km})\end{array}$ & & & & \\
\hline Cuenca 1 & Presa B-1 & 73.96 & 316.30 & 13.43 & 23.55 & 21.25 & 10.06 & 0.70 & 1.16 \\
\hline Cuenca 2 & Presa B-2 & 131.63 & 749.62 & 14.65 & 51.17 & 37.71 & 23.06 & 0.53 & 1.35 \\
\hline Cuenca 3 & Presa B-3 & 139.47 & 844.02 & 15.59 & 54.15 & 46.21 & 32.29 & 0.40 & 1.34 \\
\hline Cuenca & Nombre & $\begin{array}{l}\text { Orden de } \\
\text { corriente }\end{array}$ & $\begin{array}{l}\text { Tipos de } \\
\text { corriente }\end{array}$ & $\begin{array}{c}\text { Densidad de } \\
\text { drenaje }\end{array}$ & $\begin{array}{c}\text { media } \\
(\mathrm{msnm})\end{array}$ & $\begin{array}{c}\text { cuenca } \\
(\%)\end{array}$ & $\begin{array}{c}\text { mayor } \\
(\mathrm{msnm})\end{array}$ & $\begin{array}{c}\text { menor } \\
(\mathrm{msnm})\end{array}$ & $\begin{array}{c}\text { Cauce Princi- } \\
\text { pal }(\%)\end{array}$ \\
\hline Cuenca 1 & Presa B-1 & 3 & perennes & 0.215 & 4753.74 & 0.143 & 4725 & 4510 & 0.0101 \\
\hline Cuenca 2 & Presa B-2 & 4 & perennes & 0.293 & 4630.59 & 0.123 & 4725 & 4330 & 0.0105 \\
\hline Cuenca 3 & Presa B-3 & 4 & perennes & 0.335 & 4604.65 & 0.126 & 4725 & 4280 & 0.0096 \\
\hline
\end{tabular}
la zona de Ananea no se han encontrado pozos artificiales para la explotación de agua subterráneas. La cuenca alta del río Grande en la zona de Ananea comprende de $9.14 \mathrm{~km}^{2}$ de área de nevados.

Tabla 2: Parámetros geomorfológicos de la cuenca alta del río Grande. 
Tabla 3: Valores de la precipitación promedio mensual en las estaciones meteorológicas con influencia en la cuenca alta del río Grande durante el periodo 1964-2009. Fuente: Elaboración propia con datos del SENAMHI.

\begin{tabular}{ccccccccccccc}
\hline Estación & $\mathrm{E}$ & $\mathrm{F}$ & $\mathrm{M}$ & $\mathrm{A}$ & $\mathrm{M}$ & $\mathrm{J}$ & $\mathrm{J}$ & $\mathrm{A}$ & $\mathrm{S}$ & $\mathrm{O}$ & $\mathrm{N}$ & $\mathrm{D}$ \\
\hline Ananea & 124.9 & 103.0 & 92.0 & 45.3 & 14.8 & 7.3 & 5.8 & 15.1 & 24.5 & 43.7 & 60.9 & 93.2 \\
Putina & 146.2 & 106.3 & 102.9 & 49.5 & 9.7 & 5.2 & 4.4 & 8.5 & 28.3 & 49.7 & 70.3 & 96.8 \\
Cuyo Cuyo & 147.1 & 127.8 & 116.4 & 62.1 & 31.6 & 18.6 & 18.6 & 31.1 & 46.6 & 64.0 & 68.2 & 118.1 \\
\hline
\end{tabular}

Los parámetros geomorfológicos de la cuenca del río Grande en la zona de Ananea se muestran en la tabla 2.

Respecto a la escorrentía, la cuenca del río Grande en la zona de Ananea no cuenta con estaciones hidrométricas e hidrográficas que registren los caudales aportantes por esta cuenca. Con respecto a la pluviometría en la tabla 3, se presenta la precipitación mensual de las estaciones meteorológicas cercanas al área de estudio en la cuenca alta del río Grande.

Los histogramas de la información de precipitación media mensual histórica para las estaciones de Ananea y Putina, cercanas al área de estudio, se muestra en las figuras 2 y 3 .

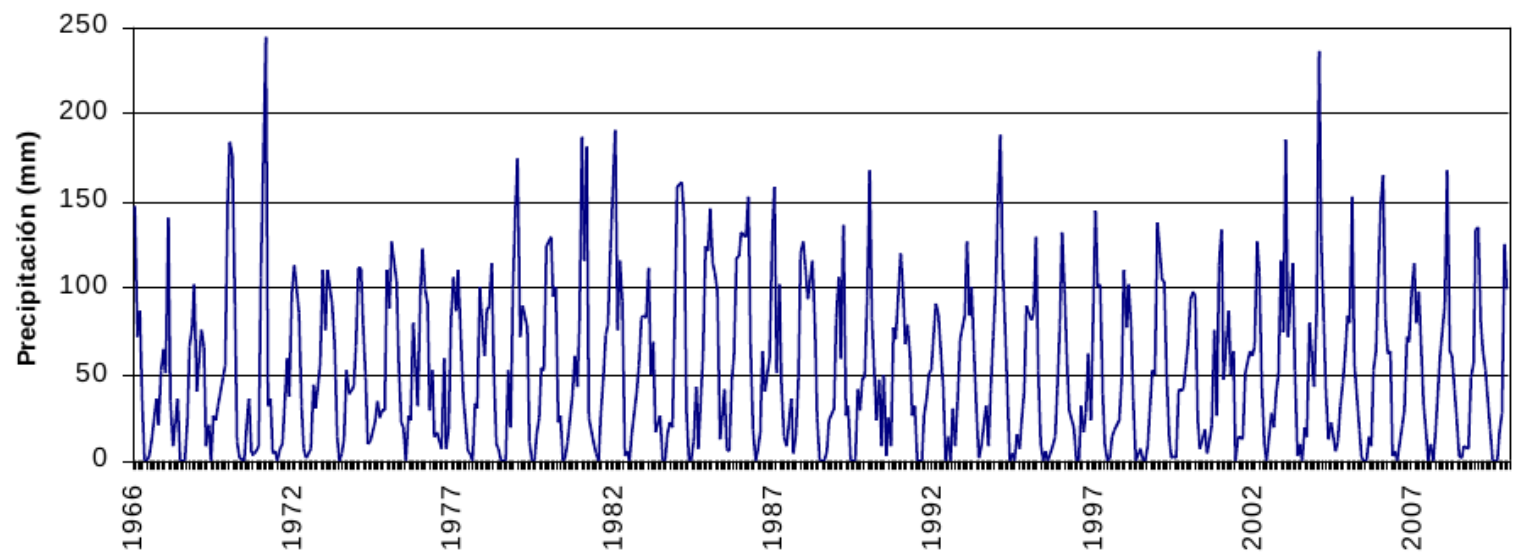

Tiempo (Años)

Figura 2: Histograma de la precipitación de la estación de Ananea en el periodo 1965-2009.

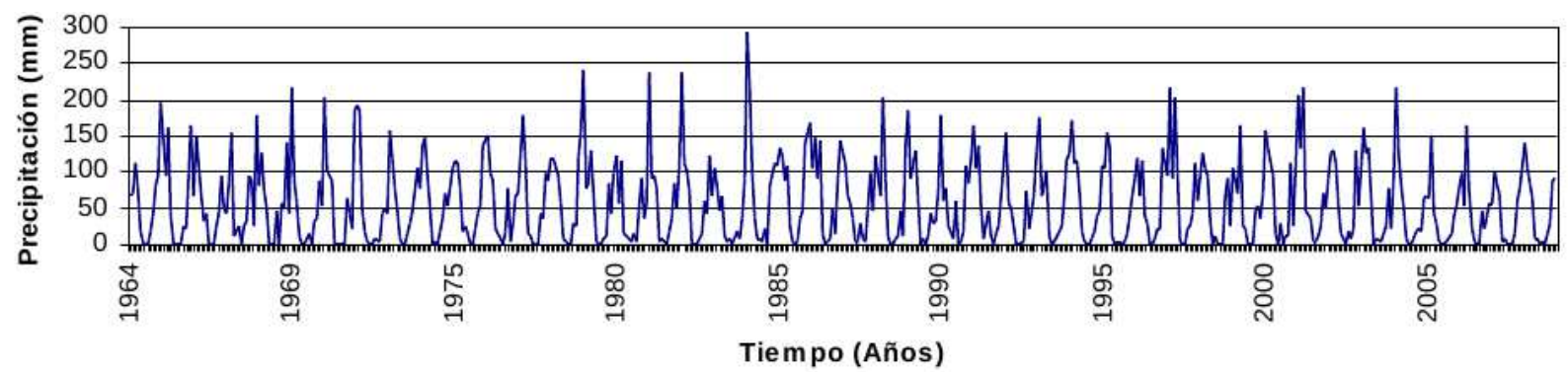

Figura 3: Histograma de la precipitación de la estación de Putina en el periodo 1965-2009.

Análisis de la consistencia de la información de la precipitación

El análisis de la información de la precipitación se ha tomado en cuenta la localización, altitud, período de registro y evaluaciones de campo. Los datos faltantes del registro de precipitación de la estación de Ananea, correspondiente al período 1969-1970, y los datos faltantes de la precipitación en la estación de Putina en los años 1974 y 2006, fueron completados mediante el uso del modelo HEC-4 del Hydrologic Engineering Center, U.S. Army Corps of Engineers [7]. 
Con esta información de la precipitación completada se efectuó el análisis de la Curva de Doble Masa, CDM, entre los datos de la estación de Ananea y Putina, apreciándose que la recta de doble masa tiene una buena linealidad, comprobando con ello la consistencia y la homogeneidad de la información de precipitación de la estación Ananea, ver figura 4.

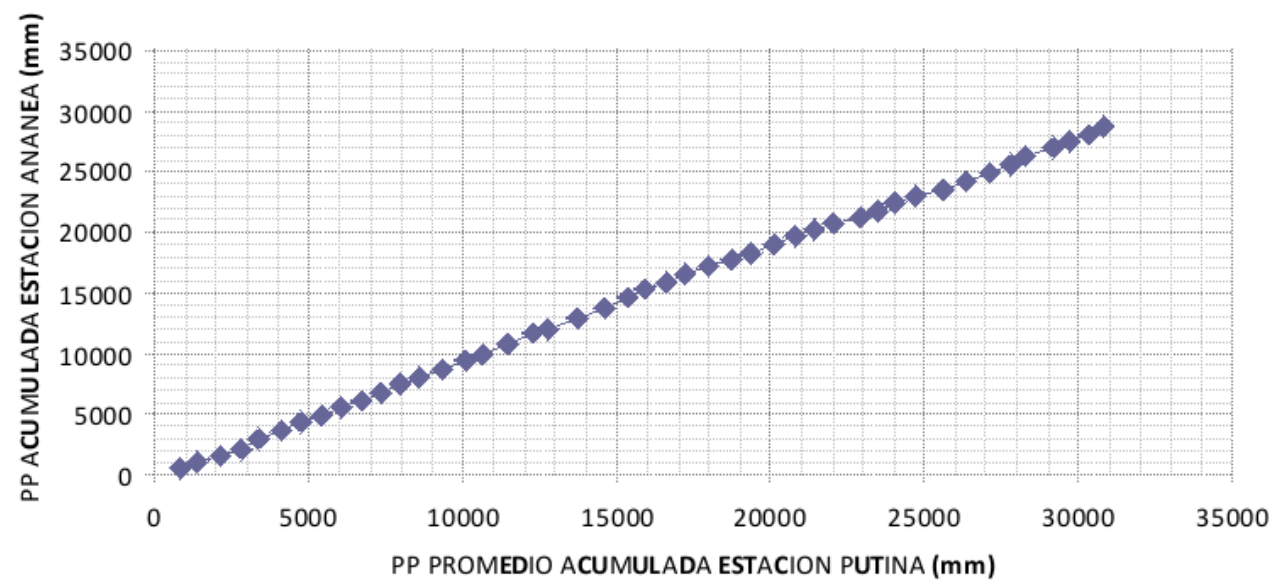

Figura 4: Se muestra la curva de doble masa de estación de Ananea vs la estación de Putina.

\section{Resultados}

Habiendo escasez de estaciones pluviométricas en el área de estudio, y considerando que la ubicación de la estación de Ananea está, aproximadamente, en la parte media de la cuenca del río Grande donde se ubica la Presa B-1, se asume que la precipitación media de la cuenca es, también, de la Presa B-1.

Determinamos el coeficiente de escorrentía, considerando que la temperatura media anual en Ananea es $4.3^{\circ} \mathrm{C}$ y tiene una precipitación media anual de 638.2 $\mathrm{mm} /$ año, ver tabla 6 . Reemplazando estos valores en las Ecs. (1), (2) y (3), se obtiene $L=411.5, D=351.03$ $\mathrm{mm} /$ año y $C=0.45$. Este valor de coeficiente de escorrentía de 0.45 es utilizado en los cálculos hidrológicos.

La determinación de los caudales medios mensuales en la cuenca alta del río Grande en la zona de Ananea donde se encuentra la cuenca de la Presa B-1, se realiza mediante la información disponible de la precipitación total mensual de $638.2 \mathrm{~mm}$ (tabla 6), el área de la cuenca de la Presa B-1 de $316.30 \mathrm{~km}^{2}$, la retención de la cuenca de Presa B-1 de $20.5 \mathrm{~mm} / \mathrm{año} \mathrm{y} \mathrm{el}$ coeficiente de agotamiento $a=0.0115$ obtenido de la Ec.(4) . a) Con el registro de la precipitación total mensual completa del periodo 1965-2009 de la estación de Ananea se calcula la respectiva precipitación efectiva por el método de la FAO, ver tabla 1, los resultados obtenidos se muestran en la tabla 4 para el período 1965-2009. b) Se calcula la retención producida por las lagunas, los pantanos y la napa freática de la cuenca de la Presa B-1, ver tabla 5. c) Se calcula el gasto de la retención $G_{i}$ a partir del mes de abril hasta el mes de octubre (7 meses), determinando (I) la relación entre la descarga del mes actual y del mes anterior, Ec. (5) y remplazando valores de $a=0.0115$ y $t=30$, se obtiene $g_{0}=0.70836$, (II) la relación de descarga del mes actual y del mes anterior para la estación seca se obtiene usando la Ec.(6). Los resultados se muestran en la tabla 6, y (III) para el cálculo del gasto de la retención, de los meses de la estación seca (abril-setiembre) se aplica la Ec. (7). Los resultados se muestran en la tabla 6 . d) Se calcula el abastecimiento o la alimentación de la retención, que se presenta en la estación lluviosa (octubre-marzo) con la Ec. (8). Los resultados se muestran en la tabla 6. e) Generación de caudales mensuales para el año promedio con la Ec.(9). Los resultados se muestran en la tabla 6. f) Con los resultados expuestos en la tabla 6 , se efectúa la regresión múltiple entre el $Q_{t}$, el $Q_{(t-1)}$ y la $P_{E t}$, (tabla 7 ), determinándose los coeficientes de regresión, el error estándar y el coeficiente de correlación, y se genera los números aleatorios con distribución normal con media cero y variancia igual a 1 . g) Con los resultados de los ítems $e), f), g$ ) se procede a la generación de los caudales medios mensuales mediante la Ec.(10). Reemplazando valores de las variables en esta ecuación se tiene los coeficientes del modelo, $Q_{t}=5.1696+0.3242 Q_{t-1}+0.4803 P E_{t}+2.3284 Z_{t i}$.

En la tabla 08, se presentan los valores de los caudales generados en $\mathrm{m}^{3} / \mathrm{s}$ para un periodo de 45 años (1965-2009), utilizando el modelo antes mencionado. De estos resultados se deduce que el volumen promedio total anual aportado por la cuenca de la Presa B-1 es de 100.87 Mmc. 
Tabla 4: Resumen de la precipitación efectiva obtenida con el método de la FAO en el periodo 1965-2009.

\begin{tabular}{cccccccccccccc}
\hline Año & Ene & Feb & Mar & Abr & May & Jun & Jul & Ago & Set & Oct & Nov & Dic & Tot \\
\hline Prom & 75.68 & 58.35 & 50.21 & 18.05 & 2.93 & 3.21 & 1.21 & 3.20 & 7.31 & 16.60 & 28.57 & 52.03 & 317.34 \\
Máx & 164.04 & 170.28 & 120.60 & 53.16 & 27.56 & 82.49 & 18.73 & 16.28 & 35.78 & 38.60 & 75.24 & 96.36 & 488.78 \\
Mín & 14.06 & 17.72 & 3.74 & 0.00 & 0.00 & 0.00 & 0.00 & 0.00 & 0.00 & 0.00 & 0.00 & 0.00 & 206.72 \\
\hline
\end{tabular}

Tabla 5: Retención debido a las lagunas, pantanos y la napa freática de la Cuenca Presa B-1.

\begin{tabular}{lccc}
\hline Descripción & $\begin{array}{c}\text { Área } \\
\left(\mathrm{km}^{2}\right)\end{array}$ & $\begin{array}{c}\text { Lámina específica } \\
(\mathrm{mm} / \mathrm{año})\end{array}$ & $\begin{array}{c}\text { Volumen total } \\
\left(\mathrm{Mm}^{3}\right)\end{array}$ \\
\hline Napa freática & 10 & 250 & 2.500 \\
Lagunas y pantanos & 27.13 & 500 & 13.565 \\
Nevados & 9.14 & 500 & 4.570 \\
\hline Total & 46.27 & & 20.635 \\
\hline \multicolumn{2}{c}{$R_{i}=$ Volumen Total $\left(\mathrm{m}^{3}\right) /$ Área Cuenca $\left(\mathrm{m}^{2}\right)=65.24 \mathrm{~mm} /$ año. }
\end{tabular}

Tabla 6: Caudal de aportación por año promedio de la cuenca de recepción de la Presa B-1.

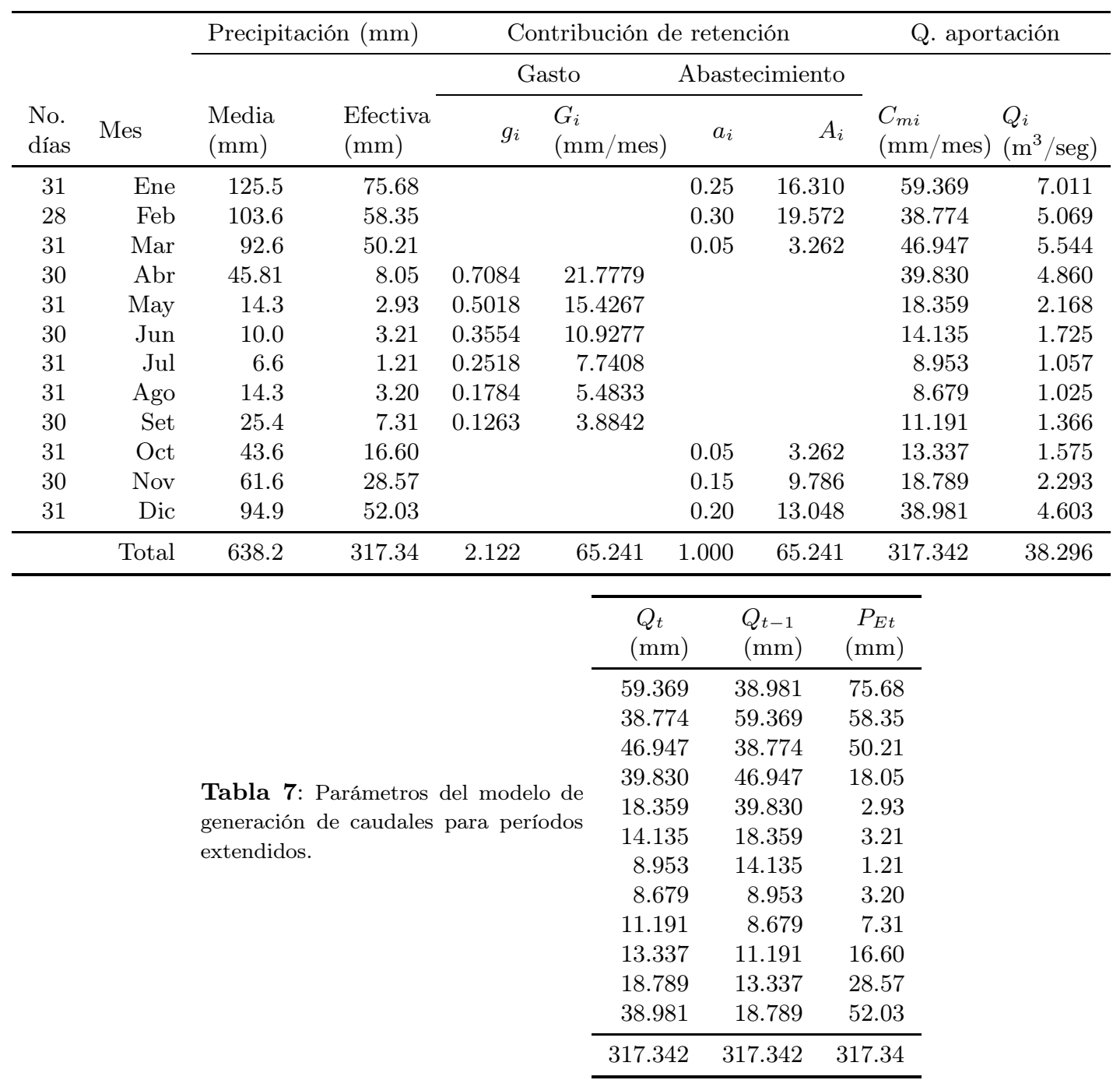


Tabla 8: Caudales mensuales generados en el cauce de la Presa B-1 en el río Grande de la zona de Ananea $\left(\mathrm{m}^{3} / \mathrm{s}\right)$.

\begin{tabular}{|c|c|c|c|c|c|c|c|c|c|c|c|c|c|}
\hline Año & Ene & Feb & Mar & Abr & May & Jun & Jul & Ago & Set & Oct & Nov & Dic & Prom \\
\hline 1965 & 7.52 & 5.37 & 4.62 & 2.31 & 1.28 & 0.80 & 0.67 & 1.17 & 1.54 & 1.38 & 1.92 & 3.02 & 2.63 \\
\hline 1966 & 2.61 & 7.22 & 2.89 & 2.09 & 2.03 & 1.41 & 1.47 & 0.97 & 1.00 & 2.82 & 3.35 & 4.92 & 2.73 \\
\hline 1967 & 3.04 & 4.38 & 3.67 & 2.15 & 1.29 & 0.98 & 1.88 & 1.55 & 1.53 & 1.98 & 3.13 & 7.58 & 2.76 \\
\hline 1968 & 9.83 & 11.69 & 6.55 & 2.99 & 1.84 & 1.52 & 1.42 & 1.70 & 1.10 & 1.15 & 1.29 & 0.87 & 3.50 \\
\hline 1969 & 5.53 & 4.74 & 4.22 & 3.96 & 1.91 & 5.78 & 3.28 & 1.44 & 1.24 & 1.01 & 3.13 & 5.64 & 3.49 \\
\hline 1970 & 7.34 & 9.86 & 8.45 & 4.55 & 1.99 & 1.14 & 0.93 & 1.27 & 3.55 & 2.99 & 2.11 & 5.46 & 4.14 \\
\hline 1971 & 5.91 & 13.25 & 4.38 & 2.93 & 1.02 & 0.52 & 1.00 & 1.15 & 1.26 & 2.44 & 2.28 & 4.47 & 3.38 \\
\hline 1972 & 5.71 & 6.00 & 5.07 & 2.05 & 1.08 & 0.52 & 0.49 & 1.70 & 1.99 & 2.24 & 2.01 & 4.47 & 2.78 \\
\hline 1973 & 4.25 & 6.42 & 4.85 & 3.85 & 1.82 & 1.15 & 0.92 & 0.72 & 1.64 & 2.00 & 2.47 & 2.91 & 2.75 \\
\hline 1974 & 5.14 & 6.46 & 4.66 & 3.59 & 2.17 & 1.19 & 1.00 & 1.84 & 1.44 & 1.81 & 1.99 & 4.47 & 2.98 \\
\hline 1975 & 4.84 & 7.33 & 5.94 & 4.46 & 2.01 & 1.21 & 1.08 & 1.50 & 1.38 & 3.28 & 1.78 & 3.76 & 3.21 \\
\hline 1976 & 6.03 & 6.58 & 5.27 & 2.50 & 2.61 & 1.57 & 1.45 & 1.04 & 2.69 & 1.55 & 2.04 & 3.10 & 3.04 \\
\hline 1977 & 5.34 & 5.30 & 5.44 & 3.01 & 2.09 & 1.49 & 1.29 & 1.25 & 1.57 & 1.26 & 4.17 & 3.66 & 2.99 \\
\hline 1978 & 4.15 & 4.72 & 5.80 & 3.66 & 1.63 & 1.30 & 0.64 & 0.47 & 2.14 & 1.27 & 4.07 & 6.59 & 3.04 \\
\hline 1979 & 9.10 & 5.52 & 4.74 & 4.21 & 1.80 & 1.18 & 0.82 & 0.81 & 1.23 & 2.37 & 2.66 & 5.61 & 3.34 \\
\hline 1980 & 6.89 & 6.11 & 5.20 & 2.53 & 1.51 & 1.25 & 0.94 & 1.00 & 1.25 & 2.19 & 2.08 & 3.80 & 2.90 \\
\hline 1981 & 8.85 & 8.22 & 10.12 & 4.51 & 2.27 & 1.56 & 1.43 & 1.28 & 1.83 & 3.18 & 3.96 & 5.92 & 4.43 \\
\hline 1982 & 10.12 & 6.44 & 6.58 & 5.57 & 2.37 & 1.51 & 0.86 & 1.18 & 2.18 & 3.04 & 4.26 & 4.50 & 4.05 \\
\hline 1983 & 4.50 & 5.49 & 3.37 & 3.95 & 2.40 & 1.86 & 0.88 & 0.30 & 0.97 & 1.12 & 1.27 & 2.53 & 2.39 \\
\hline 1984 & 7.39 & 10.64 & 8.86 & 4.18 & 1.86 & 1.24 & 1.15 & 1.72 & 0.96 & 2.86 & 5.88 & 6.36 & 4.42 \\
\hline 1985 & 8.07 & 7.65 & 6.13 & 6.19 & 2.75 & 2.12 & 1.60 & 1.00 & 2.30 & 2.71 & 5.72 & 6.59 & 4.40 \\
\hline 1986 & 7.00 & 7.72 & 8.02 & 5.09 & 2.15 & 1.40 & 1.36 & 1.28 & 2.13 & 2.49 & 3.41 & 6.44 & 4.04 \\
\hline 1987 & 8.68 & 5.09 & 4.61 & 3.27 & 1.61 & 1.27 & 1.18 & 0.92 & 1.38 & 2.19 & 6.03 & 6.82 & 3.59 \\
\hline 1988 & 6.45 & 5.86 & 6.59 & 4.64 & 2.15 & 0.99 & 0.95 & 0.91 & 1.37 & 2.01 & 1.67 & 3.00 & 3.05 \\
\hline 1989 & 4.58 & 3.99 & 6.39 & 3.88 & 2.45 & 1.27 & 0.89 & 2.35 & 2.03 & 3.02 & 2.71 & 4.40 & 3.16 \\
\hline 1990 & 8.26 & 6.39 & 3.15 & 2.33 & 1.76 & 2.13 & 1.66 & 1.63 & 1.55 & 2.87 & 3.64 & 5.49 & 3.41 \\
\hline 1991 & 5.09 & 4.27 & 3.94 & 3.87 & 2.26 & 1.93 & 1.04 & 1.07 & 1.33 & 1.85 & 2.65 & 2.22 & 2.63 \\
\hline 1992 & 3.67 & 5.03 & 4.37 & 2.40 & 0.81 & 1.03 & 0.73 & 1.80 & 1.08 & 1.52 & 3.20 & 4.26 & 2.49 \\
\hline 1993 & 6.39 & 5.61 & 5.13 & 3.93 & 2.47 & 1.21 & 0.68 & 1.48 & 1.46 & 1.80 & 3.18 & 4.94 & 3.19 \\
\hline 1994 & 7.75 & 11.72 & 7.87 & 5.35 & 2.18 & 1.45 & 1.23 & 1.01 & 1.47 & 1.31 & 2.03 & 5.01 & 4.03 \\
\hline 1995 & 4.09 & 4.47 & 6.27 & 4.40 & 2.34 & 1.78 & 1.00 & 0.85 & 1.39 & 1.06 & 1.93 & 3.13 & 2.73 \\
\hline 1996 & 5.64 & 5.88 & 3.71 & 1.75 & 1.57 & 1.47 & 1.49 & 1.36 & 1.13 & 1.08 & 2.43 & 1.71 & 2.44 \\
\hline 1997 & 5.97 & 5.99 & 5.62 & 3.39 & 1.33 & 0.99 & 0.78 & 0.81 & 1.31 & 1.11 & 2.48 & 4.60 & 2.87 \\
\hline 1998 & 4.34 & 5.50 & 4.38 & 2.79 & 1.68 & 1.57 & 0.60 & 0.26 & 0.45 & 1.53 & 4.46 & 3.27 & 2.57 \\
\hline 1999 & 6.62 & 6.30 & 5.64 & 3.41 & 1.57 & 1.04 & 1.03 & 1.13 & 2.01 & 2.34 & 2.61 & 3.18 & 3.07 \\
\hline 2000 & 4.56 & 5.73 & 5.09 & 2.60 & 1.64 & 1.68 & 1.01 & 1.19 & 1.02 & 2.67 & 1.71 & 4.76 & 2.80 \\
\hline 2001 & 6.54 & 4.35 & 4.64 & 3.57 & 2.98 & 2.04 & 1.80 & 1.25 & 1.29 & 2.21 & 2.92 & 3.04 & 3.05 \\
\hline 2002 & 3.31 & 6.48 & 5.75 & 3.31 & 1.85 & 1.12 & 1.63 & 1.43 & 2.32 & 2.35 & 5.24 & 4.47 & 3.27 \\
\hline 2003 & 9.35 & 5.84 & 5.79 & 3.95 & 1.28 & 1.29 & 1.42 & 0.67 & 0.52 & 3.32 & 2.49 & 4.28 & 3.35 \\
\hline 2004 & 11.48 & 9.06 & 5.82 & 3.93 & 1.94 & 1.29 & 0.83 & 0.88 & 1.88 & 2.63 & 3.29 & 3.57 & 3.88 \\
\hline 2005 & 4.33 & 8.72 & 4.85 & 2.43 & 1.22 & 1.43 & 0.65 & 0.51 & 0.91 & 2.42 & 3.14 & 6.99 & 3.13 \\
\hline 2006 & 8.87 & 6.68 & 4.08 & 3.86 & 1.88 & 1.45 & 1.00 & 1.17 & 1.83 & 2.79 & 3.72 & 4.71 & 3.50 \\
\hline 2007 & 5.82 & 5.27 & 4.88 & 2.84 & 1.92 & 1.60 & 1.17 & 1.36 & 0.80 & 1.36 & 2.89 & 5.17 & 2.92 \\
\hline 2008 & 8.22 & 5.15 & 3.67 & 2.57 & 1.43 & 2.10 & 1.38 & 1.28 & 1.52 & 2.34 & 2.62 & 5.85 & 3.18 \\
\hline 2009 & 7.29 & 5.34 & 3.38 & 3.12 & 1.70 & 1.52 & 1.09 & 1.12 & 1.01 & 1.17 & 4.79 & 5.21 & 3.06 \\
\hline Máx & 11.48 & 13.25 & 10.12 & 6.19 & 2.98 & 5.78 & 3.28 & 2.35 & 3.55 & 3.32 & 6.03 & 7.58 & 4.43 \\
\hline Mín & 2.61 & 3.99 & 2.89 & 1.75 & 0.81 & 0.52 & 0.49 & 0.26 & 0.45 & 1.01 & 1.27 & 0.87 & 2.39 \\
\hline Prom & 6.37 & 6.57 & 5.34 & 3.51 & 1.87 & 1.47 & 1.15 & 1.17 & 1.51 & 2.09 & 3.04 & 4.51 & 3.22 \\
\hline Desvest. & 2.06 & 2.10 & 1.54 & 1.01 & 0.47 & 0.75 & 0.47 & 0.42 & 0.57 & 0.70 & 1.19 & 1.48 & 0.54 \\
\hline
\end{tabular}




\section{Conclusiones}

a) La cuenca del río Grande en la zona de Ananea, cuenta con una extensión de $316.3 \mathrm{Km}^{2}$ hasta el punto de la boquilla de la Presa B-1 y una altitud media de 4753.74 m.s.n.m., y nace en los nevados de Ananea y Ccorhuani y de las lagunas al pie de los cerros Huincho y Suchuita. b) Se ha comprobado la eficiencia de la metodología para determinar el rendimiento hídrico superficial a partir de los datos de la precipitación mensual en cuencas que carecen de registros de caudales medios mensuales y con poca información hidrometeorológica, como es el caso de la cuenca alta del río Grande, zona de Ananea, que ha permitido determinar el recurso hídrico a ser depositado en el embalse de la Presa B-1. c) La Presa B-1 tiene por finalidad el de almacenar y retener los sedimentos producidos por la mineria formal e informal de la explotación de oro que se viene desarrollando actualmente en esta cuenca. $d$ ) Se ha determinado una serie de caudales medios mensuales de 45 años, período 1965-2009, a partir de la cual se ha determinado que el caudal mínimo en el punto de la boquilla de la Presa B1 es de $0.26 \mathrm{~m}^{3} / \mathrm{s}$ y el máximo es de $13.25 \mathrm{~m}^{3} / \mathrm{s}$, siendo el potencial hídrico anual en este punto de 100.07 Mmc. e) Los resultados preliminares de este trabajo han sido presentados en el Taller de Investigación de Física 2011, llevado a cabo el 3-5 de noviembre del 2011.

\section{Referencias}

[1] Loayza, Ch \& Galloso, Implicancias Ambientales por la Actividad Minera de la Zona de Ananea en la Cuenca del río Ramis, INGEMMET, Boletín No. 5, Serie E: Minería, 104 p. (2008).

[2] Pérez H. \& Castro S. Reconocimiento de las Actividades Mineras y Metalúrgicas en la Cordillera Suroriental, San Antonio de Putina - Puno. INGEMMET. (2003)

[3] Programa Nacional de Pequeñas Irrigaciones. Generación de Caudales Mensuales en la Sierra Peruana, Ed. UNALM, Lima (1997).
[4] Salas, J. D., J. W. Delleur, V. Yevyevich, and W.L. Lane, Applied Modeling od Hydrologic Times Seies, WWP, Littleton, Colorado (2002).

[5] Linsley Ray K. Warter Resources Engineering, Ed. Mc. Graw-Hill Interamericana, (1979).

[6] Sistemas, Agua y Energía S.A. Planeamiento Hidroenergético de la Cuenca del Río Ramis, (2008).

[7] U.S. Army Corps of Engineers, HEC-4. Monthly Streamflow Simulation, (1971). 